\title{
Physical and chemical properties of restructured sweet potato stick from three sweet potato cultivars
}

\begin{abstract}
Restructured Sweetpotato Stick (RSS) was developed in attempt to overcome the lack of sweetpotato availability during off season. Moreover, RSS was produced aimed to control of the adequate qualities caused by the variation characteristics of sweetpotato. The making of RSS from 3 sweetpotato varieties was to study physicochemical and sensory attributes of the final product. RSS was produced using three sweetpotato (SP) varieties with different flesh colour namely White, Yellow and Orange. Samples, including raw materials, intermediate and final products were analyzed for physicochemical attributes. White and Orange cultivars judged generated the good quality RSS. A similar hardness and shearing force of RSS made of White and Orange was found. Proximate compositions on dry base (db) were: protein 3.59 and $2.74 \%$, fat 21.44 and $35.91 \%$, carbohydrate 71.83 and $59.19 \%$; and ash 3.13 and $2.17 \%$, respectively. White cultivar produced the RSS having yellow bright colour, high firmness and low fat content, whereas Orange cultivar generated RSS with bright orange colour, medium firmness but high fat content. RSS made from White and Orange cultivars were preferred with sensory score above the average. Result from this study illustrates that White and Orange sweetpotato cultivars can be used to make a convenient restructured product (RSS).
\end{abstract}

Keyword: RSS; Texture; Physicochemical; Sensory 